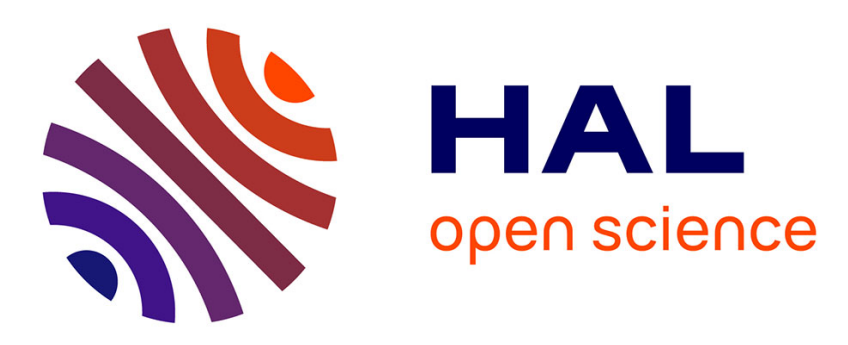

\title{
Découverte d'hermelles fossiles dans le Messinien de San Miguel de Salinas (Annélides du Miocène Supérieur du Sud-est de l'Espagne
}

\author{
Manuel Garcin, Daniel Vachard
}

\section{> To cite this version:}

Manuel Garcin, Daniel Vachard. Découverte d'hermelles fossiles dans le Messinien de San Miguel de Salinas (Annélides du Miocène Supérieur du Sud-est de l'Espagne. Geobios, 1987, 20 (3), pp.407-414. 10.1016/S0016-6995(87)80050-5 . hal-00562386v2

\section{HAL Id: hal-00562386}

https://hal-brgm.archives-ouvertes.fr/hal-00562386v2

Submitted on 15 Nov 2012

HAL is a multi-disciplinary open access archive for the deposit and dissemination of scientific research documents, whether they are published or not. The documents may come from teaching and research institutions in France or abroad, or from public or private research centers.
L'archive ouverte pluridisciplinaire HAL, est destinée au dépôt et à la diffusion de documents scientifiques de niveau recherche, publiés ou non, émanant des établissements d'enseignement et de recherche français ou étrangers, des laboratoires publics ou privés. 


\title{
DÉCOUVERTE D'HERMELLES FOSSILES DANS LE MESSINIEN DE SAN MIGUEL DE SALINAS (ANNÉLIDES DU MIOCÈNE SUPÉRIEUR \\ DU SUD-EST DE L'ESPAGNE)
}

\author{
par \\ MANUEL GARCIN * \& DANIEL VACHARD *
}

\section{RÉSUMÉ}

Le Messinien du Sud-Est de l'Espagne livre de petits biohermes composés de tubes millimétriques, étroitement serrés, à paroi calcaire agglutinante, qui possèdent beaucoup de points communs avec les constructions actuelles de Sabellariidae. Ce sont probablement les premiers fossiles indiscutables de ce groupe.

\begin{abstract}
Small organic bioherms composed of closely packed, carbonate-walled tubes, probably constructed by Sabellariid Worms have been discovered in the " preevaporitic » Messinian strata from Southeastern Spain. These building-rocks are similar to Recent bioherms in east-central peninsular Florida (USA) and in Mont-Saint-Michel Bay (Western France).

These Messinian Sabellariid-reefs are associated with various intertidalites : hardened surfaces, beach rocks, beds with wave ripples and flaser structures, Cardium in living position. Thus fossil Sabellariidreefs are growing in lower intertidal area.
\end{abstract}

RESUMEN

En la cuenca de San Miguel de Salinas (Alicante) se descubrieron arrecifes de Gusanos Anélidos Poliquetos Sabelarias semejantes a los biohermos conocidos en las costas actuales de Florida y del Oeste de Francia. El yacimiento fosilífero, de edad mesiniana, está incluido en una sedimentación de tipo intertídalo con figuras de corrientes, litificación rapida y varios macrofósiles característicos de estas ambientes como los Cardium.

* Institut de Géologie I.G.A.L., 21 rue d'Assas, 75270 Paris Cedex 06.

Geobios, $\mathbf{n}^{\circ} 20$, fasc. 3

p. 407-414, 1 fig., 1 pl.

Lyon, juin 1987 


\section{INTRODUCTION}

Situé au SE de l'Espagne, à l'extrémité des Cordillères Bétiques (fig. 1A), le bassin de San Miguel comporte quatre groupes de dépôts messiniens (Montenat 1977 ; Garcin 1987 ; fig. 1B) :

(1) des marnes à Foraminifères planctoniques de la zone à Conomiozea-Mediterranea en continuité parfaite avec les marnes tortoniennes de la zone à Suterae-Exserta ;

(2) des marnes à intercalations monotones de grès jaunes ;

(3) des faciès diversifiés dits du « Messinien préévaporitique » constitués de dépôts marneux, de bancs gréseux parfois dus à des écoulements gravitaires, de constructions récifales à Porites et Algues rouges, de niveaux d'estran à Hermelles qui feront l'objet de cette note, de marnes lagunaires à Charophytes et ripple-marks ;

(4) des faciès du "Messinien évaporitique » rythmés par des séquences où se succèdent : (a) des stromatolithes et des calcaires rognonneux à feuilles de végétaux terrestres; (b) des calcaires blancs oolithiques et sableux à ciment de ménisque et clinostratifications parfois en chevron (herring-bone) indiquant des dépôts de plages ; (c) des sables jaunes à langues de progradation correspondant probablement à des « fan-deltas "; (d) des marnes de décantation azoïques ou à rares organismes planctoniques et benthiques ; (e) des gypses sélénites toujours développés en fin de séquence.

Les profondeurs de ces ensembles sont variables. Les marnes à Foraminifères planctoniques se déposent vers 400-500 $\mathrm{m}$ de fond dans l'ensemble des bassins bétiques (Brimaud \& Vachard 1985) ; les alternances de grès et de marnes ont encore cette profondeur. Le « préévaporitique » voit sa paléogéographie et ses profondeurs se modifier : le large bassin fait place à une cuvette dont le fond se situe vers 100-200 $\mathrm{m}$, et où récifs et estrans marquent les bordures subémergentes. Le Messinien évaporitique se développe dans la même cuvette ; les gypses sous-marins se forment par $50 \mathrm{~m}$ de fond environ (Garcin 1987). L'importante variation de niveau à la base du « préévaporitique » correspond à la baisse eustatique de 250 m enregistrée sur les courbes de Vail et alii (1977).

\section{LE GISEMENT À HERMELLES}

La coupe ayant livré les Hermelles affleure à quelque distance à l'Est du village de Puerto-Rebate. Elle se situe sur le flanc d'une petite falaise, où les prises de photographies et d'échantillons sont difficiles.

La série montre les niveaux suivants de bas en haut (fig. 1C) :

(a) $40 \mathrm{~cm}$ de calcaires sableux jaûnatres faiylement bioclastiques ;

(b) $20 \mathrm{~cm}$ de grès calcaires coquilliers à nombreux tests de Pectinidés et d'Ostréidés ; le sommet est une surface durcie rubéfiée ;

(c) $40 \mathrm{~cm}$ de grès calcaires riches en Ostrea offreti KILIAN forées par des Cliones. Dans ce banc se rencontrent divers lithoclastes et les premiers fragments de tubes d'Annélides. Au sommet on trouve des Cardium en position de vie (pl. 1, fig. 3), puis une surface durcie creusée de petites poches et encroûtées par des Serpules ; (d) $1 \mathrm{~m}$ de constructions à Annélides (pl. 1, fig. 1-2). Les biohermes (ou biolithosores au sens de Lucas 1959) ont une forme en " champignon " avec des bouquets de tubes plus ou moins évasés au sommet. Les espaces entre les biohermes sont remplis par un calcaire sableux bioclastique montrant de nombreux fragments de constructions à Annélides. La lentille à biolithosores s'étend sur $200 \mathrm{~m}$ de long environ ;

(e) $70 \mathrm{~cm}$ de calcaires sableux beiges, presque azoïques, présentant dans la partie inférieure, de nombreuses figures de courants : laminations horizontales (laminated sands), rides de vagues (wave ripples), structures madrées (flaser structures). Le sommet montre une surface durcie ravinée ;

(f) base d'une construction récifale (troisième récif ou R3 de Garcin 1987) à Rhodophycées, Porites et nombreux lithoclastes.

La lithologie, l'agencement séquentiel régional, les rides de courants, les surfaces durcies ravinées et les 


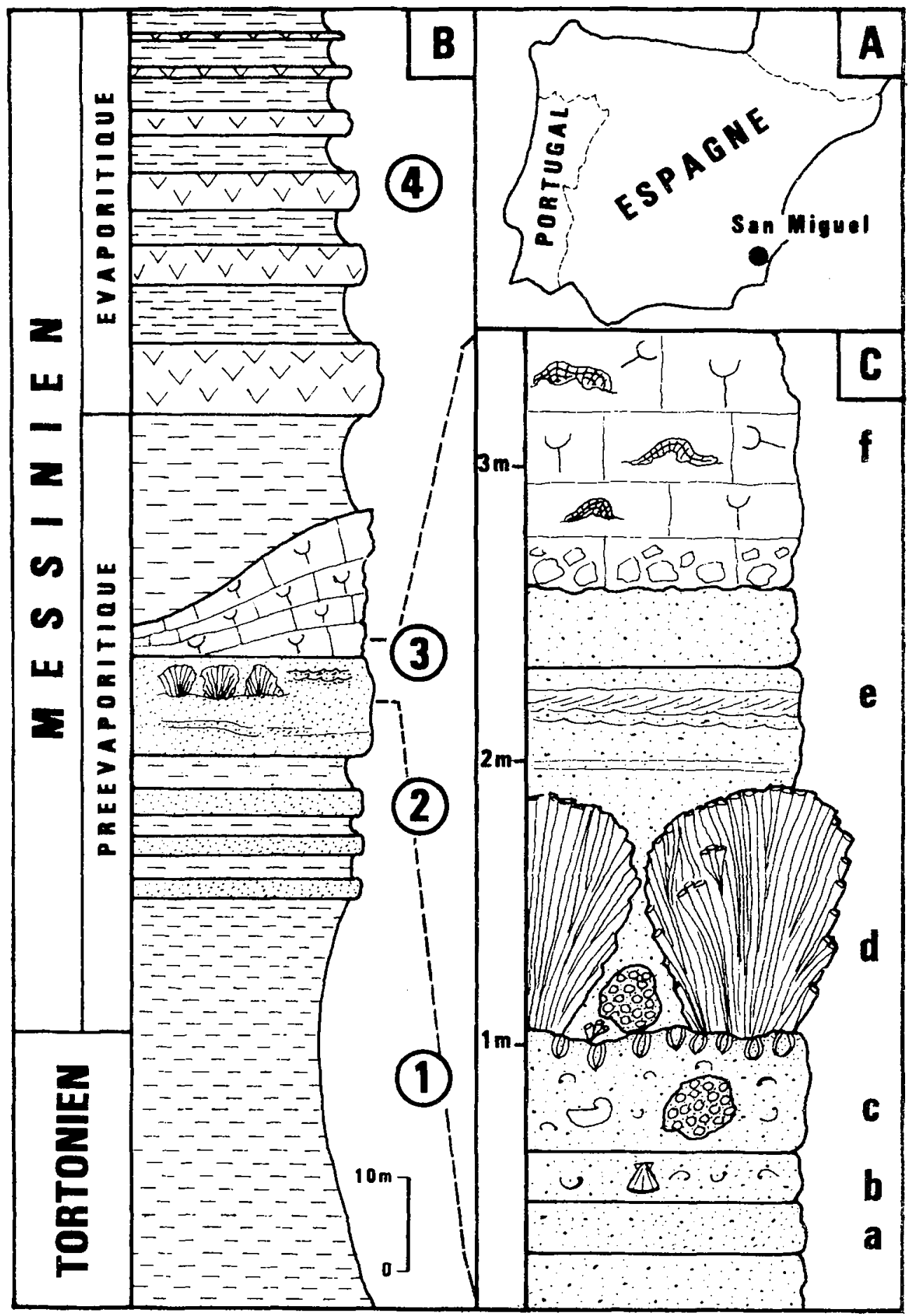

Fig. I - A : Localisation de la région étudiée.

B : Séries schématiques du Messinien (explications dans le texte).

C : Succession des terrains à Hermelles (explications dans le texte).

A : Localization of the studied area.

$B$ : Sketch of the Messinian strata (cxplanations in text).

$C$ : Succession of the beds with Sabellariid reef (explanations in text). 
Cardium en position de vie plaident pour une sédimentation d'estran et donc de domaine intertidal. Les microfaciès montrent des cimentations de «beach rocks », voisines de celles décrites en Méditerranée à l'Holocène par Alexandersson (1972).

\section{PRÉCISIONS SUR LES TUBES D’ANNÉLIDES}

Les « champignons » ont une hauteur de 60 à $80 \mathrm{~cm}$ et une largeur maximale de $60 \mathrm{~cm}$. Les tubes ont une section circulaire; leur diamètre externe est de 5 à 7 $\mathrm{mm}$ et leur diamètre interne moyen de $3 \mathrm{~mm}$. L'agglutinat est formé de tests de Lamellibranches disposés obliquement et imbriqués comme les tuiles d'un toit (pl. 1, fig. 4). Il s'agit presque toujours de valves dissociées brisées. Lorsqu'elles sont complètes, leur charnière est vers l'avant et leur concavité vers l'extérieur (pl. 1, fig. 4). Plus rarement on trouve des coquilles bivalves (pl. 1, fig. 6). Divers Foraminifères sont agglutinés dans les parois ; les uns contemporains : Elphidium, Ammonia et Triloculina; les autres remaniés de niveaux allant du Crétacé supérieur au Miocène inférieur : Globotruncana, Nummulites (pl. 1, fig. 5), Discocyclina, Alveolina, Nephrolepidina, Miogypsina.

La nature du ciment reliant l'agglutinat n'a pu être déterminée pour l'instant. Le remplissage des tubes, plus fin, est généralement constitué de micrite sableuse à rares Foraminifères planctoniques (pl. 1, fig. 5).

\section{COMPARAISON AVEC LES SABELLARIIDAE ACTUELS}

Les Sabellariidae actuels et leurs constructions sont bien connus sur les côtes atlantiques de l'Europe et des Etats-Unis, notamment dans la baie de Bourgneuf (Gruet 1971), la baie du Mont-Saint-Michel (Lucas 1959 ; Mathieu 1967 ; Lang et alii 1973 ; Caline et alii 1982 ; Gruet 1972, 1982, 1986) et sur les côtes de Floride (Multer \& Milliman 1967 ; Gram 1968 ; Kirtley \& Tanner 1968).

Les principales espèces de France et de Floride, Sabellaria alveolata (LINNÉ) et Phragmatopoma lapidosa KINGBERG, produisent des biohermes assez épais, résistants à l'action des vagues, dans la partie inférieure de la zone intertidale, dans les baies ou sur les côtes rocheuses peu ou moyennement battues. Il y a donc identité de milieu de vie entre formes messiniennes et actuelles.

Aspect des constructions et dimensions des tubes sont similaires. Il existe des constructions en champignons dans la baie de Bourgneuf et du Mont-SaintMichel (Gruet 1971, 1972), ainsi que des « barrières » et des « platiers ». La hauteur varie de $70 \mathrm{~cm}$ (Lucas 1959) à 1,50 m (Lang et alii 1973). La topographie générale des ensembles construits est très fluctuante (Gruet 1986). Les tubes des Hermelles actuelles ont des sections circulaires de 5 à $10 \mathrm{~mm}$ de diamètre et une paroi épaisse de 2 à 3 mm (Mathieu 1967).

Les Hermelles actuelles pratiquent aussi le tri des grains et ont le goût des remaniements. La granulométrie de leurs prises diffère de celle des substrats sédimentaires ; plus fine (Lucas 1959) ou plus grossière (Gruet 1982). L'agglutinat est carbonaté à $70 \%$ alors que la matrice ambiante comporte beaucoup de grains de quartz; le choix est en partie dû au ver (Gruet 1982). Les Hermelles du Mont-Saint-Michel utilisent des fragments d'Alvéolines éocènes (Mathieu 1967), de la même façon que les Hermelles miocènes font appel à un matériau remanié.

La disposition et la taille des coquilles de Lamellibranches incorporées par les Annélides miocènes présentent une ressemblance frappante avec l'agglutinat des Sabellariidae de Floride schématisé par Gram (1968) et Kirtley \& Tanner (1968) ou illustré par Multer \& Milliman (1967 : fig. 4, p. 261). 


\section{CONCLUSIONS}

Les premières Hermelles fossiles semblent avoir été découvertes ; les références précédentes à des Sabellariidés fossiles, en particulier les Skolithos, avaidnt été fortement contestées (Durant 1984), avec juste raison à notre avis.

Les milieux à Hermelles fossiles appartiennent au domaine intertidal et plutôt à l'intertidal inférieur, puisque des récifs à Porites de l'infralittoral supérieur leur succèdent dans les séquences. Comme sur les estrans actuels, Hermelles et Cardium, tous deux suspensivores, sont associés dans le Miocène.

L'agglutinat exclusivement calcaire des Hermelles miocènes semble indiquer des animaux de mers subtropicales, plus proches des formes de Floride que de celles du Mont-Saint-Michel à agglutinat plus siliciclastique. La ressemblance des récifs à Porites avec les
« cayes » de Floride, dépeints par Purser (1983), confirme cette comparaison. Dernier indice de mers chaudes, les lithifications précoces en « beach rocks » ont cimenté un substrat dur idéal pour l'implantation des Sabellariidés.

Constructions à Hermelles et récifs montrent que la salinité était normale dans les mers et sur les estrans peu de temps avant la crise messinienne. Un régime de courants et de marée renouvelait les eaux.

La difficulté de fossilisation des biohermes à Sabellariidés paraît liée à la conservation des tidalites en général et à l'extrême mobilité du paysage à Hermelles (Gruet 1986).

Des études de biosédimentologie et d'anatomie comparée se poursuivent en collaboration avec Gruet et Bodeur de Nantes (Bodeur et alii 1987).

\section{RÉFÉRENCES BIBLIOGRAPHIQUES}

ALEXANDERSSON T. (1972) - Mediterranean beachrock cementation : marine precipitation of $\mathrm{Mg}$-Calcite ; in STANLEY (D.J.) : the Mediterranean Sea, a natural sedimentation laboratory. Dowden, Hutchinson \& Ross Inc., Stroudsburg : 203-223.

BODEUR Y., GARCIN M., GRUET Y. \& VACHARD D. (1987) - Biosédimentologie des constructions à Hermelles de l'île de Noirmoutier (France) ; relations avec des équivalents fossiles découverts dans le Messinien des Cordillères Bétiques (Espagne). 8th IAS Regional Meeting of Sedimentology, Tunis : 106-107.

BRIMAUD C. \& VACHARD D. (1985) - Indications paléoécologiques fournies par les Spongiaires du Miocène supérieur d'Espagne. Bull. Mus. ntn. Hist. nat., Paris, 4, 7, C, $1: 3-11$.

CALINE B., LARSONNEUR C. \& L'HOMER A. (1982) La baie du Mont-Saint-Michel : principaux environnements sédimentaires. Livre Jubilaire Lucas, Mém. Sc. Terre, Dijon, 7 : 38-51.

DURAND J. (1984) - Ichnocénoses du grès armoricain (Ordovicien inférieur du Massif Armoricain dans leur contexte sédimentologique). Geobios, Lyon, Mém. Spéc. 8 : 183-197.
GARCIN M. (1987) - Le bassin de San Miguel de Salinas (Alicante, Espagne) ; relations entre contexte structurosédimentaire et dépôts évaporitiques et carbonatés. Thèse Doct. Univ. Orsay, n 313,297 p. (inédit).

GRAM R. (1968) - A Florida Sabellariidae reef and its effect on sediment distribution. Journ. Sedim. Petrol., Golden, 38, $3: 863-868$.

GRUET Y. (1971) - Morphologie, croissance et faune associée des récifs de Sabellaria alveolata (LINNÉ) de la Bernerie-en-Retz (Loire-Atlantique). Téthys, Marseille, 3, $2: 321-380$.

GRUET Y. (1972) - Aspects morphologiques et dynamiques de constructions de l'Annélide Polychète Sabellaria alveolata (LINNE). Rec. Trav. Inst. Pêches Marit., Nantes, 36, $2: 131-161$.

GRUET Y. (1982) - Recherches sur l'écologie des « récifs 》 d'Hermelles édifiés par l'Annélide Polychète Sabellaria alveolata (LINNÉ). Thèse Doct. Etat, Nantes, n 82-03, $238 \mathrm{p}$. (inédit).

GRUET Y. (1986) - Spatio-temporal changes of Sabellarian reefs built by the sedentary Polychaete Sabellaria alveolata (LINNÉ). Marine Ecology, Hambourg, 7, 4 : 303319. 


\section{PLANCHE 1}

Hermelles fossiles du Messinien de Puerto Rebate (bassin de San Miguel, Espagne)

Fossil Sabellariids from the Messinian of Puerto Rebate (San Miguel bjsin, Spain)

Fig. 1 - Aspect général d'une construction en champignon, fixée sur une surface durcie, et légèrement évasée vers le haut. Tubes nombreux, verticaux et obliques, remplis d'une micrite blanchâtre et avec un agglutinat beige. $\mathrm{x} 0,1$.

General view of a " mushroom »-buildup weakly tapering and attached on a hardened surface. Numerous vertical and oblique tubes filled by a whitish micrite and with a brownish agglutinate.

Fig. 2 - Biolithosore naturellement incliné permettant de voir l'aspect de surface, les extrémités circulaires des tubes et la paroi relativement épaisse. $x \mathbf{0 , 1}$.

Gently tilted biolithosore allowing to see superficial parts with circular extremities of tubes and relatively thick walls.

Fig. 3 - Niveau à Cardium en position de vie (c), surmonté par des buissons à Hermelles (h). Numérotations de terrain, 3 et 4 , correspondant aux niveaux (c) et (d) de la figure et du texte. $x$ 0,5.

Level with Cardium in living position (c) topped by Worm bushes (h). Field numbers ( 3 and 4 ) are correspol ding to levels (c) and (d) of sketch and text.

Fig. 4 - Aspect général de l'agglutinat coquillier. Le haut du tube $(\mathrm{H})$ est à droite de la photo. On remarque la structure imbriquée (i) des petits tests de Lamellibranches et une valve avec la charnière (c) tournée vers le haut et vers l'avant. Lame MG 83-92 (26). x 24.

General view of the shelly agglutinate. Upper part of the tube $(\mathrm{H})$ is in the right of the microphotograph. Noteworthy are the imbricate structure of the small Lamellibranchiata tests and a valve with hinge facing upward and forward.

Fig. 5 - Agglutinat plus varié à fragments de Nummulites remaniées (n), coquilles de Lamellibranches et débris de Bryozoaires (b). L'agglutinat se situe entre la matrice ambiante $(\mathrm{m})$ et l'intérieur du tube (t). L'agglutinat est de granulométrie plus grossière que celle de $\mathrm{m}$ et $\mathrm{t}$. Lame MG 83-92 (25). x 24.

More diversified agglutinate with reworked Nummulites fragments (n), Lamellibranchiata shells and Bryozoa remains (b). Agglutinate is located between surrounding matrix $(\mathrm{m})$ and inner part of the tube $(\mathrm{t})$. Similar granulometry for $\mathrm{m}$ and $t$, coarser for the agglutinate $\mathrm{mn}$.

Fig. 6 - Détail d'un agglutinat coquillier comportant une coquille avec ses deux valves (V), même différence entre granulométries. Lame MG 83-92 (24). x 24.

Detail of a shelly agglutinate involving a bivalved shell (V). Granulometries of agglutinate and filling are different. 

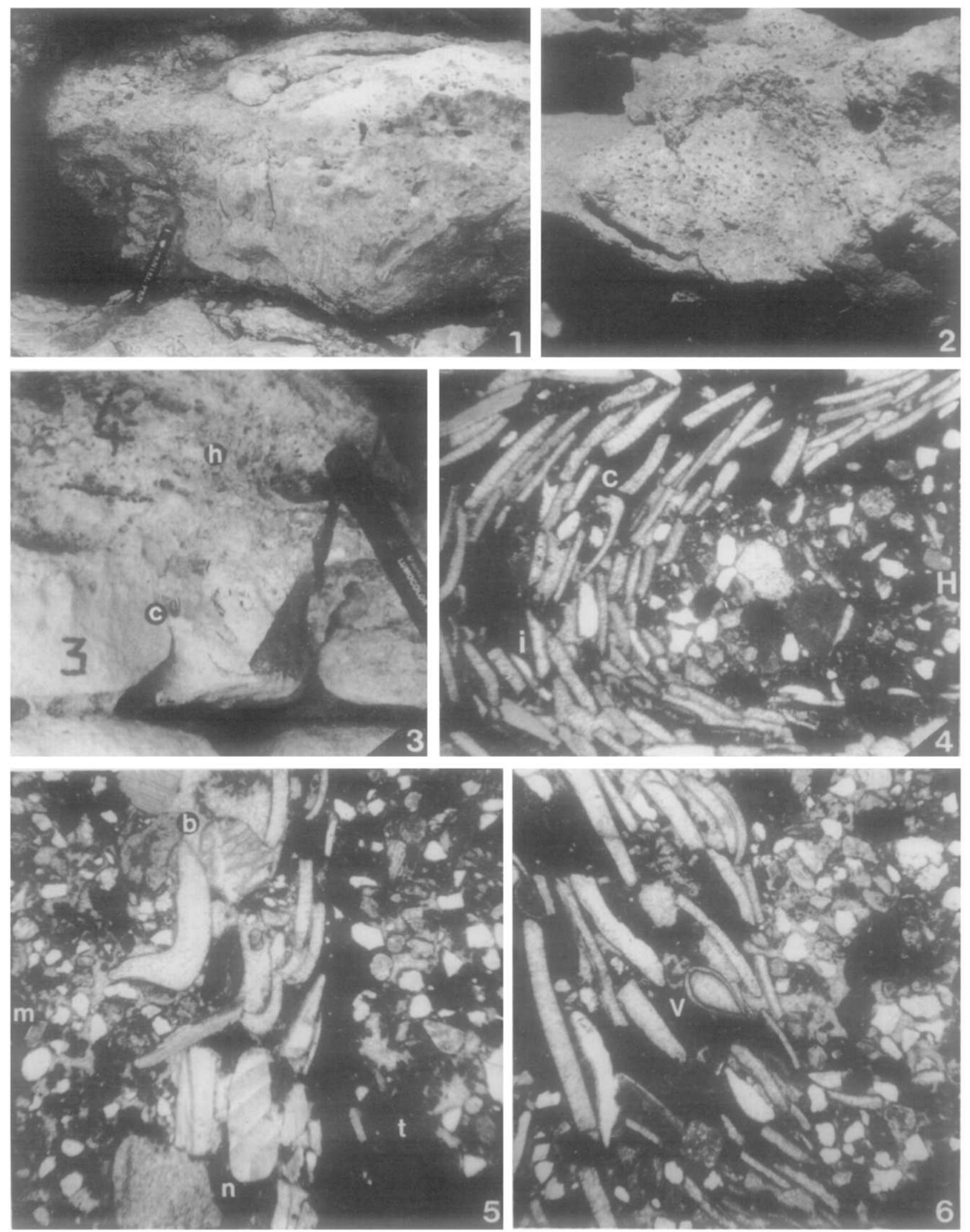
KIRTLEY D.W. \& TANNER W.F. (1968) - Sabellariid Worms : builders of major reef type. Journ. Sedim. Petrol., Golden, 38, 1 : 73-78.

LANG J., LUCAS G. \& MATHIEU R. (1973) - Le domaine benthique littoral de la baie du Mont-Saint-Michel (Manche) ; exemple d'environnement détritique marin actuel. Sciences de la Terre, Nancy, 98, $1:$ 19-78.

LUCAS G. (1959) - Deux ensembles actuels de « biolithosores » construits par des Annélides. Bull. Soc. Géol. Fr., Notes et Mém., 1, $7:$ 385-389.

MATHIEU R. (1967) - Le banc des Hermelles de la baie du Mont-Saint-Michel, bioherme à Annélides ; sédimentologie, structure et genèse; Bull. Soc. Géol. Fr, (7), 9 : 6878.
MONTENAT C. (1977) - Les bassins néogènes du Levant d'Alicante et de Murcia (Cordillères bétiques orientales, Espagne) : stratigraphie, paléogéographie et évolution dynamique. Doc. Lab. Géol. Fac. Sci. Lyon, 69, 345 p.

MULTER H.G. \& MILLIMAN J.D. (1967) - Geologic aspects of Sabellarian reefs, Southeastern Florida. Bull. Marine Science, Miami, 17, 2 : 257-267.

PURSER B.H. (1983) - Sédimentation et diagenèse des carbonates néritiques récents. Tome 2 : Les domaines de sédimentations carbonatées néritiques, application à l'interprétation des calcaires anciens, Editions Technip, Paris, 389 p.

VAIL P.R., TODD R.G. \& SANGREE J.B. (1977) - Seismic stratigraphy and global changes of sea level, part five : chronostratigraphic significance of seismic reflections. A.A.P.G. Memoir, Tulsa, 26 : 96-116. 